\title{
Marking a surgical margin for excision of a keratinocyte cancer
}

Chloe A Mutimer, Anthony J Dicker

\section{Background}

The size of the measured margin for excision of a keratinocyte cancer is often discussed; however, a technique for marking the skin is rarely described.

\section{Objective}

The aim of this article is to describe a method for marking a lesion for excision.

\section{Discussion}

The key to surgical excision of a keratinocyte cancer is the assessment of the tumour border in good light under loupe magnification and delineation using a skin marker. The surgical margin is then added around the lesion. Often an elliptical excision is performed, with lesions excised adhering to the drawn line and then closed appropriately.
THE RECENTLY REVISED Australian guidelines for managing keratinocyte cancers (formally known as non-melanoma skin cancers) includes a section on suggested clinical margins for excising these tumours. ${ }^{1}$ While the size of the measured margin is discussed, a technique for marking the skin is rarely described. Here the authors describe their method for marking out a surgical lesion for excision, which has been used and supported in several studies..$^{2-6}$

\section{Equipment}

The following equipment is recommended.

- Alcohol wipe: Cleaning the skin before any surgical drawing makes it easier to apply marks to the skin. An alcoholbased solution will evaporate within seconds, while an aqueous-based wash may cause the pen markings to run.

- Bright light: Good illumination of the lesion is essential to define the clinical edges of a lesion.

- Magnification: A simple surgical loupe enhances the ability to correctly identify the clinical edge of the lesion.

- Dermatoscope: This is an extension of the role of magnification and can help further define the clinical edge. ${ }^{7}$

- Codman marker: These surgical pens are available in sterilised packs with a ruler.

\section{Technique}

The following outlines an example for basal cell carcinoma (BCC) removal; margins can be adjusted for squamous cell carcinoma (SCC) removal.
1. Wipe the skin with an alcohol wipe. Rubbing a BCC can cause a flush of the lesion to assist in identifying the clinical margins.

2. Stretching the skin can greatly assist in identifying the edge of a lesion, particularly for a BCC. Dermatoscopic examination of the lesion before marking with a Codman marker can help identify extensions of tumour.

3. Using bright lighting, stretch and magnification, dot around the outer margin of the lesion using the Codman marker, placing the dots on the adjacent normal appearing skin (Figure 1).

4. Connect the dots to create one continuous line around the lesion border. Fill in and thicken the line so that it has a diameter of $2-3 \mathrm{~mm}$ (Figure 2). Add extra circles so that the distance from the inner edge of the inner circle and the outer edge of the outer circle is the required clinical margin. Alternatively, an outer line with a margin of 2-3 $\mathrm{mm}$ from the lesion's border can be drawn.

5. Examine and palpate the area to identify the natural direction of closure. The direction of closure may be indicated by Langer's lines, biodynamic excisional skin tension (BEST) lines, normal skin creases or junctions of cosmetic units (Figure 3).

6. When excising the lesion, cut on the outside of the pen markings with the lesion itself excised as a circle, then the ellipses formed excised afterwards (Figure 4). 
7. The method of wound closure depends on the size of the lesion, with direct closure the preferred method. Flap repairs or skin grafts are options for larger wounds.

\section{Surgical margins}

The surgical margin should aim to meet the widely accepted incomplete excision rate, in which histopathological involvement of margins is seen in $<5 \%$ of excisions. ${ }^{8}$ Margin involvement is judged by histological assessment, with

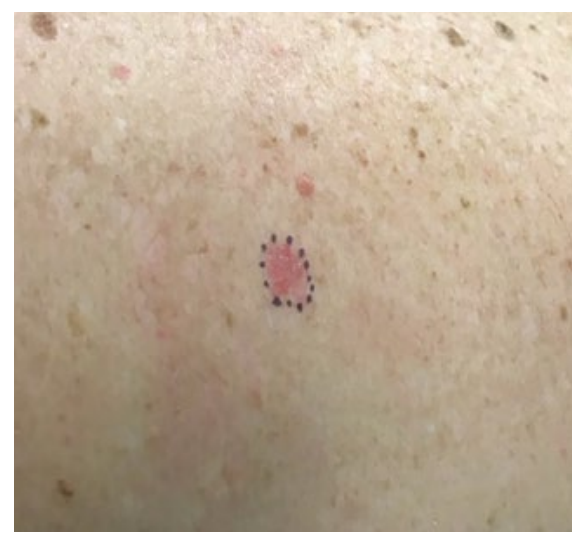

Figure 1. Basal cell carcinoma with outer edges of lesion marked with a Codman marker

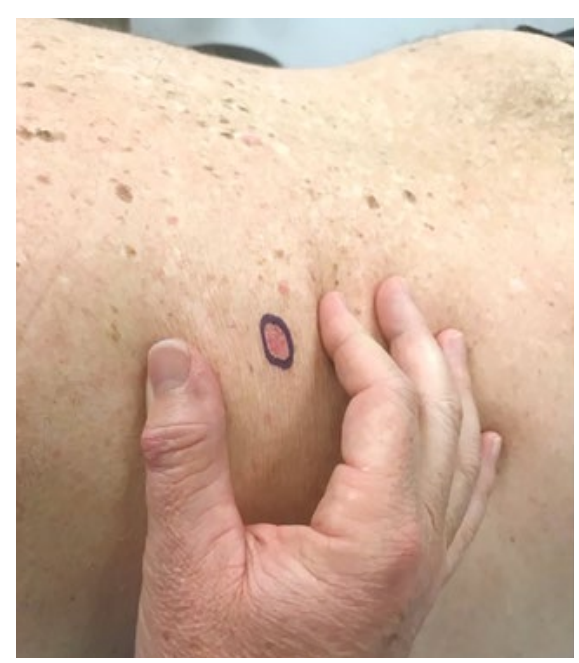

Figure 3. Skin placed under tension to determine the direction of closure tumour being evident at the edge of the histological specimen.

Recommendations for surgical margins are shown in Table 1. In relation to SCC, surgical margins of $4 \mathrm{~mm}$ are generally recommended for low-risk lesions and $6 \mathrm{~mm}$ for high-risk lesions. ${ }^{9}$ An adequate surgical margin for BCCs has been widely debated. Cancer Council Australia's clinical guidelines suggest $2-3 \mathrm{~mm}$ surgical margins for low-risk tumours and margins $>5 \mathrm{~mm}$ for high-risk tumours. ${ }^{1}$ Most research has occurred in tertiary referral centres, where excisions were

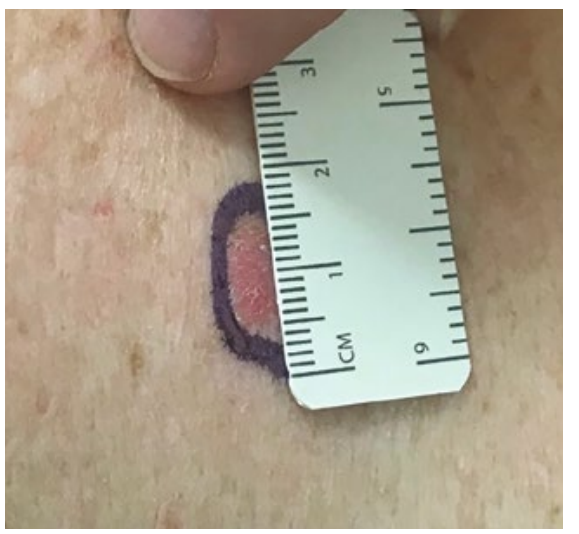

Figure 2. Dots around the margin connected to form a continuous line, measured to have a diameter of 2-3 mm

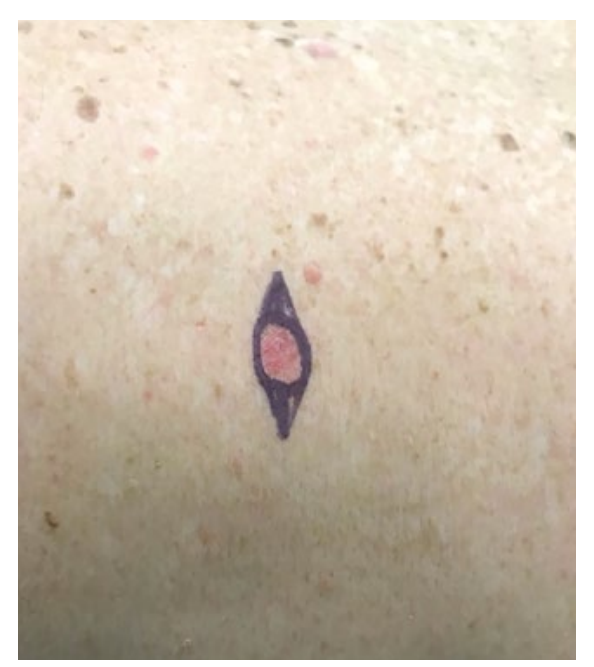

Figure 4. Ellipse drawn around the lesion for elliptical excision; the outer edge of the markings is the line of excision performed by dermatologists or plastic surgeons. There may be a bias for the population treated in these studies, as patients referred to these centres are more likely to have high-risk or difficult-to-treat lesions, compared with those treated in primary centres.

Ultimately, selection of an appropriate surgical margin should aim to achieve a balance between rates of incomplete excision of the tumour and normal tissue sacrifice, which can affect functional and cosmetic outcomes, a factor that is often important to patients.

\section{When to consider referral}

Most BCCs can be managed in a primary care centre. However, it is recommended that referral to a dermatologist or plastic surgeon for surgical excision is considered on the basis of individual patient factors such as tumour location (head and neck), histological subtype (infiltrating or sclerosing), size ( $>2 \mathrm{~cm}$ in diameter) and patient preference. Other treatments - such as Mohs micrographic surgery, curettage and cautery, cryotherapy, topical therapies (such as imiquimod) or radiotherapy can be considered on a case-by-case basis and compared with surgery, with the aim of achieving the best outcome for the patient.

High-risk features of SCC include size $>20 \mathrm{~mm}$, tumour depth $>4 \mathrm{~mm}$, recurrent lesion, high-risk anatomic location (head and neck), perineural or lymphovascular invasion, poorly differentiated subtype or immunosuppression. ${ }^{1}$ Patients with high-risk features should be referred to a specialist or multidisciplinary team for management. Other management options include Mohs micrographic surgery, curettage and cautery, cryotherapy, topical medications and photodynamic therapy. ${ }^{10}$ If a tumour is not appropriate for excision because of patient factors (eg not being fit for surgery) or further locoregional control is sought, referral to a radiation oncologist can also be considered. Referral for medical oncology review should be sought in the case of metastatic SCC.

The Australian guidelines suggest that recurrent or incompletely excised 
Table 1. Suggested margins depending on features of the lesion ${ }^{1}$

\begin{tabular}{lrr}
\hline Low-risk lesion & $\begin{array}{r}\text { Margins for basal cell } \\
\text { carcinoma excision }\end{array}$ & $\begin{array}{r}\text { Margins for squamous cell } \\
\text { carcinoma excision }\end{array}$ \\
\hline High-risk lesion & $2-3 \mathrm{~mm}$ & $4 \mathrm{~mm}$ \\
\hline
\end{tabular}

lesions be referred for specialist management. ${ }^{1}$ In regard to incomplete excision, management options include further re-excision, topical treatments or monitoring depending on a number of lesion factors including whether the lateral or deep margins were involved. ${ }^{11,12}$

\section{Further management}

All patients treated for keratinocyte carcinomas require follow-up. This can either be undertaken by their general practitioner or non-GP specialist, and is needed to assess for evidence of recurrence, metastasis and new primary skin cancers. Patient education regarding the prevention of skin cancer is also crucial.

\section{Key points}

- The keratinocyte cancer guidelines provide good advice for clinicians undertaking surgical excision of skin tumours.
- Bright light and good magnification are important for determining the edges of

- Referral should be considered based on individual factors, such as tumour location, size and patient preference.

\section{Authors}

Chloe A Mutimer BBMed, MD (Distinct), Medical Registrar, Alfred Hospital, Vic; Australian Skin Cancer Clinics, Vic

Anthony J Dicker MBBS, PhD, Skin Cancer Physician,

Australian Skin Cancer Clinics, Vic; University of

Queensland, Qld

Competing interests: None.

Funding: None.

Provenance and peer review: Not commissioned, externally peer reviewed.

Correspondence to:

c.mutimer@alfred.org.au

\section{References}

1. Cancer Council Australia Keratinocyte Cancers Guideline Working Party. Draft clinical practice guidelines for keratinocyte cancer. Sydney, NSW Cancer Council Australia, 2019. Available at https:// wiki.cancer.org.au/australia/Guidelines:Keratinocyte carcinoma [Accessed 5 February 2021].

2. Lalloo MT, Sood S. Head and neck basal cell carcinoma: Treatment using a 2-mm clinical the tumour. excision margin. Clin Otolaryngol Allied Sci 2000;25(5):370-73. doi: 10.1046/j.13652273.2000.00376.x

3. Kimyai-Asadi A, Alam M, Goldberg LH, Peterson SR, Silapunt S, Jih MH. Efficacy of narrow-margin excision of well-demarcated primary facial basal cell carcinomas. J Am Acad Dermatol 2005;53(3):464-68. doi: 10.1016/j. jaad.2005.03.038.

4. Bisson MA, Dunkin CS, Suvarna SK, Griffiths RW. Do plastic surgeons resect basal cell carcinomas too widely? A prospective study comparing surgical and histological margins. Br J Plast Surg 2002;55(4):293-97. doi: 10.1054/bjps.2002.3829.

5. Thomas DJ, King AR, Peat BG. Excision margins for nonmelanotic skin cancer. Plast Reconstr Surg 2003;112(1):57-63. doi: 10.1097/01. PRS.0000067479.77859.31.

6. Griffiths R, Suvarna SK, Stone J. Basal cell carcinoma histological clearance margins: An analysis of 1539 conventionally excised tumours. Wider still and deeper? J Plast Reconstr Aesthet Surg 2007;60(1):41-47. doi: 10.1016/j. bjps.2006.06.009.

7. Lallas A, Apalla Z, loannides D, et al. Dermoscopy in the diagnosis and management of basal cell carcinoma. Future Oncol 2015;11(22):2975-84. doi: 10.2217/fon.15.193.

8. Wolf DJ, Zitelli JA. Surgical margins for basal cell carcinoma. Arch Dermatol 1987;123(3):340-44.

9. Nahhas AF, Scarbrough CA, Trotter S. A review of the global guidelines on surgical margins for nonmelanoma skin cancers. J Clin Aesthet Dermatol 2017;10(4):37-46.

10. Kallini JR, Hamed N, Khachemoune A. Squamous cell carcinoma of the skin: Epidemiology, classification, management, and novel trends. Int $J$ Dermatol 2015;54(2):130-40. doi: 10.1111/ijd.12553.

11. Gulleth $Y$, Goldberg N, Silverman RP, Gastman BR. What is the best surgical margin for a basal cell carcinoma: A meta-analysis of the literature. Plast Reconstr Surg 2010;126(4):1222-31. doi: 10.1097/ PRS.0b013e3181ea450d.

12. Bovill ES, Banwell PE. Re-excision of incompletely excised cutaneous squamous cell carcinoma: Histological findings influence prognosis. J Plast Reconstr Aesthet Surg 2012;65(10):1390-95. doi: 10.1016/j.bjps.2012.04.031. 